Original Research Paper

\title{
Extracting the Potential Features of Digital Panoramic Radiograph Images by Combining Radio Morphometry Index, Texture Analysis, and Morphological Features
}

\author{
Enny Itje Sela, Sutarman \\ Universitas Teknologi Yogyakarta, Yogyakarta, Indonesia
}

\author{
Article history \\ Received: 01-09-2017 \\ Revised: 02-11-2017 \\ Accepted: 17-11-2017 \\ Corresponding Author: \\ Enny Itje Sela \\ Universitas Teknologi \\ Yogyakarta; \\ Yogyakarta, Indonesia \\ Email: ennysela@yahoo.com
}

\begin{abstract}
Osteoporosis is a type of disease that is not easily detected visually. It contributes to bone fracture and so early diagnosis is particularly important to prevent bone fracture. An integrated approach for extraction of cortical and trabecular bone on the digital panoramic radiograph (DPR) images was proposed to screen osteoporosis. We performed radio morphometry index (RMI), texture analysis, and morphology analysis to extract the features of DPR images. Then, the extracted features were further applied to decision tree technique which lead to obtain potential or significant features about osteoporosis. An automated classifier was developed based on Learning Vector Quantization (LVQ) to differentiate between normal and osteoporotic class. In this study, seven major features playing significant role in the osteoporosis identification. For testing purpose, the accuracy of decision tree technique resulted $96,77 \%$ and the accuracy of LVQ was $80 \%$.
\end{abstract}

Keywords: Decision tree, LVQ, Morphology analysis, Radio morphometry index, Texture analysis

\section{Introduction}

Osteoporosis is still a problem in public health, especially in developing countries. Osteoporosis is a type of disease that is not easily detected visually. People with osteoporosis will be aware of osteoporosis at suddenly they had a fracture through any accident. That is why early osteoporosis's screening is a useful effort to prevent it (Sela et al., 2015). Early examination of osteoporosis by utilizing dental images is very helpful, since osteoporosis examination using DEXA is still expensive and not all hospitals provide this facility (Levine, 2016; Sela and Widyaningrum, 2015).

According (Sela et al., 2014), when the body needs calcium, it will be taken from the jaw bones, first. That is why the jaw bone suffered more fragility than others. The dental radiograph images have been widely explored for osteoporosis screening. The dental image itself consists of panoramic and periapical images. The parts of the panoramic image used for the screening of osteoporosis are the cortical bone and trabecular bone, whereas the dental periapical image only utilizes the trabecular bone.
Therefore, the panoramic images have greater potential for osteoporosis detection than periapical images.

There have been several studies to develop the method of osteoporosis screening using the dental radiographs images (Sela et al., 2013; Widyaningrum et al., 2012; Rao et al., 2011; Milliuniene et al., 2008). On previous studies, the digital panoramic radiograph (DPR) images were explored separately on the cortical bone section using radio morphometry index (RMI) measurement, whereas the trabecular bone was explored using texture analysis.

In this study, the DPR images are explored more deeply by combining radio morphometry index, texture analysis, and morphology analysis. Furthermore, the selected features will be process using neural network Learning Vector Quantization (LVQ) for osteoporosis identification.

\section{Materials}

This study involves the Javanese ethnic of menopausal women in Indonesia, and the subject's age 
were between 40 and 57 years $(50.0 \pm 4.3)$. The dental panoramic radiographs were taken from previous study. It consisted of 62 images in the JPG format with a size of $1024 \times 1024$. Assessment of BMD on femoral neck and lumbar spine carried out from the Department of Radiology, Dr. Sardjito Hospital using DEXA densitometer. The specifications of DEXA are $76 \mathrm{kV}$, $1.5 \mathrm{~mA}$, during $1 \mathrm{~min} 14 \mathrm{~s}$ (femoral), and $1 \mathrm{~min} 27 \mathrm{~s}$ (spine). We classified subjects into one of two groups: normal $(\mathrm{N})$ and osteoporotic (OP). We have 62 images on normal class (33 images) and the rest was osteoporotic class (29 images).

\section{Methods}

This research proposed model is shown in Fig. 1.

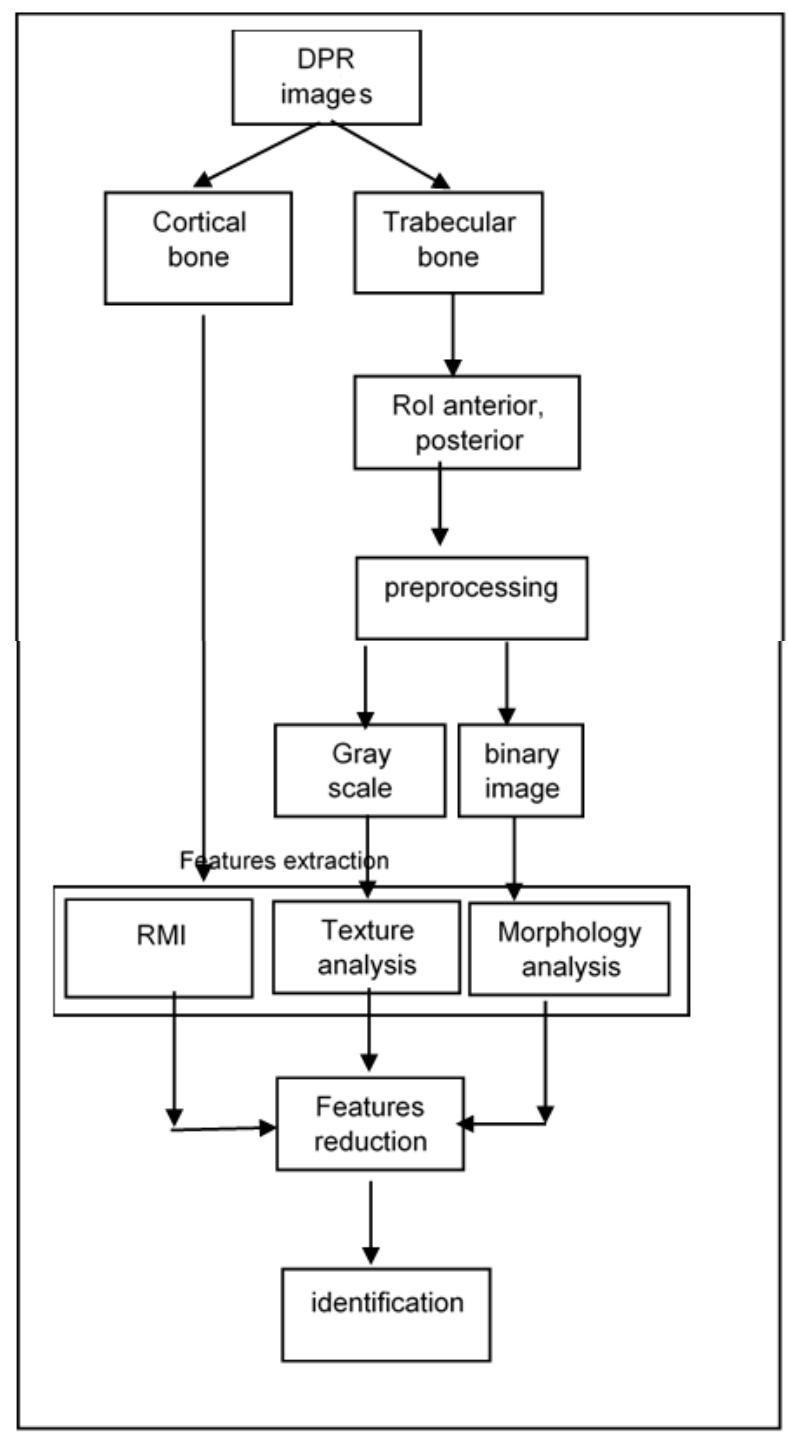

Fig. 1: Research Model

\section{Preprocessing}

The preprocessing was performed to improve the quality of RoIs. Preprocessing in this study was also used to prepare the RoIs image on trabecular bone in order to obtain texture and morphology features. From a DPR image on trabecular bone, we cropped three RoIs: the anterior RoI (28x64 pixels), the left and right posterior RoIs (64x64 pixels). Cropping process was performed manually using Adobe Photoshop software. Each RoI were transformed into a gray scale image so that texture analysis can be obtained. In addition, the RoI images were also transformed into binary image for morphological analysis. There were several stages of preprocessing to get the binary image, which were reducing noise with tophat-bothat filtering, transforming ROI to gray scale image, and thresholding from gray scale image (Sela et al., 2015)

\section{Features Extraction}

The normal and osteoporotic class on the DPR images could be distinguished from their RMI, texture analysis and morphology of porous features.

The measurements of RMI were performed by 2 observers within 1-week intervals using digital radiography measurement DBSWin4.5, Durr Dental R. (Widyaningrum, and Lestari, 2013). In this study, measurements of the RMI used the results of the study. From a panoramic image on the cortical bone portion can be obtained 4 RMI namely mental index (MI), panoramic mandibular index (PMI), gonion index (GI), and antegonial index (AI).

In this study, morphological analysis had been applied to the anterior, left posterior, and right posterior RoIs. Each RoI extracted 6 features based on its morphology (Sela et al., 2015; Sela and Widyaningrum, 2016) i.e. porosity(Por), small pore (JK), large pore (JB), vertical pore $(\mathrm{V})$, horizontal pore $(\mathrm{H})$, and oblique pore (M). Thus, in morphological analysis, an image would have 18 features. These features were OAntPor (porosity on anterior), OkiPor (porosity on left posterior), OkaPor (porosity on right posterior), OAntJK (small pore on anterior), OkiJK (small pore on left posterior), OKaJK (small pore on right posterior), OAntJB (large pore on anterior), OKaJB (large pore on right posterior), OKiJB (large pore on left posterior), OAnt (horizontal pore on anterior), OkiH (horizontal pore on left posterior), OkaH (horizontal pore on right posterior), OAntV (vertical pore on large posterior), OKiV (vertical pore on left posterior), $\mathrm{OKaV}$ (vertical pore on right posterior) OAntO (oblique pore on anterior), OkiO (oblique pore on large posterior), and $\mathrm{OkaO}$ (oblique pore on right posterior). The extraction features stage using the measurement of radio morphometric index, texture analysis, morphological analysis resulted 34 features. 
The texture analysis is used to obtain characteristics of objects that have a repetition pattern of the intensity value of adjacent pixels. Natural textures tend to have a uniform pattern compared to human-made textures. The methods of texture analysis are first-order statistics consist of mean, standard deviation, entropy, variance; and second order statistics consist of GLCM (GrayLevel Co-occurrence Matrix). GLCM depicts how different combinations of pixel brightness values occur in an image (Veluchamy et al., 2012; Hiremath et al., 2007). The second-order statistics measures the relationship between neighboring pixels.

In GLCM, to eliminate the tension on the image size, the element values need to be normalized. Each GLCM is normalized by the sum of all its elements to compute the co-occurrence relative frequency between gray level $i$ and $j$. Each GLCM matrix element is the probability (p) that a pixel with value $i$ will be found adjacent to a pixel of value $\mathrm{j}$ as in (1):

$$
G=\left[\begin{array}{cccc}
p(1,1) & p(1,2) & \ldots & p(1, N g) \\
p(2,1) & p(2,2) & \ldots & p(2, N g) \\
\ldots & \ldots & \ldots & \ldots \\
p(N g, 1) & p(N g, 2) & \ldots & p(N g, N g)
\end{array}\right]
$$

There are some GLCM features: angular second moment (ASM), contrast, inverse difference moment (IDM), entropy, and correlation. In this research, four features were extracted from the GLCM matrix which were contrast, correlation, energy, and homogeneity.

Statistics properties of the image derived from GLCM in this research will be shown in (2), (3), (4) and (5). We use the following notation. $G$ is the number of gray levels. The $\mathrm{p}(\mathrm{i}, \mathrm{j})$ is the matrix element obtained through GLCM. The $\mu$ is the mean value of $p(i, j)$. Symbols of $\mu 1, \mu 2, \sigma 1$ and $\sigma 2$ are the means and standard deviations of $\mathrm{Pi}$ and $\mathrm{P} 2$ respectively.

We created two gray-level co-occurrence matrices (GLCM) from the image, specifying different offsets [2 $0 ; 0$ 2]. Each row in this p-by-2 matrix defines a single relative position in [row column] format. The first row of the matrix is [2 0$]$ means each pixel is compared to the pixel 2 rows down, 0 columns over (i.e. in the same column). The second row [0 2] indicates that each pixel is also compared to the pixel 0 rows away (i.e. in the same row) and 2 columns. Thus, each pixel is compared against two of its neighbor pixels: the pixel 2 columns to the right, and the pixel 2 rows down. The pairs formed by both relationships are used to increment the appropriate pixels in the output image.

Energy (E) is also known as angular second moment (ASM). It was performed by the sum of squared elements in the GLCM as in (2). It values between 0 and 1. Energy is 1 for a constant image:
$E=\sum_{i, j} p(i, j)^{2}$

Contrast (C) shows the size of the spread (moment of inertia) elements of the image matrix. If the location is far from the main diagonal, then the contrast is large. Visually, the value of contrast is a measure of variation between the gray degrees of an area:

$C=\sum_{i, j}^{G-1}(i-j)^{2} p(i, j)$

Correlation (Corr) shows the size of the linear dependence of the grayness of the image so that it can give a show of the linear structure in the image. Correlation is 1 or -1 for a perfectly positively or negatively correlated image. The equation is in (4):

Cor $=\sum_{i, j} \frac{\left(i-\mu_{i}\right)\left(j-\mu_{j}\right) p(i, j)}{\sigma_{i} \sigma_{j}}$

Homogeneity $(\mathrm{H})$ denotes the distribution of matrix's elements in the GLCM to the GLCM diagonal, as in (5). The value of $\mathrm{H}$ is between 0 and 1 . Homogeneity is 1 for a diagonal GLCM:

$H=\sum_{i, j} \frac{p(i, j)}{1+|i-j|}$

The extraction process using texture analysis, had been applied to the anterior, posterior, left and right posterior RoIs. In each RoI were extracted 4 features based on analysis texture: entropy, contrast, correlation, homogeneity. Thus, on texture analysis, an image will have 12 features. These features are Aa (entropy on anterior), $\mathrm{AKa}$ (entropy on right posterior), $\mathrm{AK}$ (anterior contrast on left), $\mathrm{BKa}$ (contrast on right posterior), $\mathrm{BKi}$ (contrast on left posterior), $\mathrm{Ca}$ (correlation on anterior), $\mathrm{CKa}$ (correlation on right posterior), $\mathrm{CKi}$ (correlation on left posterior), Da (homogeneity on anterior), DKa (homogeneity on right posterior), and DKi (homogeneity on left posterior).

\section{Reduction Features}

Reduction features have two potential benefits of: (a) reducing time of training and (b) defying the curse of dimensionality so that prediction performance could be improved. Selecting the most relevant features is usually suboptimal for building a predictor. Decision tree is a method of the embedded selection features. A decision tree is a tree in which each branch node represents a choice between several alternatives. Decision tree generate the rules starts with a root node. From this node, users split each node recursively decision tree 
learning algorithm (Vasudevan, 2014; Sutarman et al., 2017; Mantzaris et al., 2010). Leaf nodes represent the normal or osteoporosis category. We used J48 algorithm to build decision tree.

\section{Identification}

The method of artificial neural network (ANN) learning vector quantitation (LVQ) was conducted to train the selected features. LVQ is a method for conducting training on supervised competitive layers. This layer will automatically learn to classify the input vectors. If some input vectors have very close spacing, then the input vectors will be grouped in the same class. The distance measurement method used is Euclidian distance. In this study, the input vector on LVQ is the reduction feature while the number of classes are 2 (normal class and osteoporosis class).

\section{Results}

Based on the research, the following will be explained about the results of research. RoI images were cropped manually on the anterior trabecular bone, posterior right and left. The result of RoI can be seen in Fig. 2. While the result of preprocessing the image in the form of gray scale and binary can be seen in Fig. 3. Figure 4 shows the process of morphological analysis to obtain porous size and porous orientation in 2 classes: normal and osteoporosis. The processes are segmentation, closing operation, and filling.

On the feature extraction process on all images, with radio morphometric index, texture analysis, and morphological analysis; these features were stored in matrix $62 \times 34$. The decision tree method generated potential features by eliminating features that were considered not important. Thus, the matrix size can be reduced to $62 \times 7$. The combined of all features have the important features as demonstrated decision tree (Fig. 5) and its performance (Fig. 6). Figure 6 shows that the potential features for detecting osteoporosis using decision trees are GI, OkaH, PMI, OkiJK, OkaPor, OanJK, and $\mathrm{OkiH}$; and the most important feature is GI.

The selection results accuracy rate is $96.7742 \%$. From the confusion matrix (Fig. 6), can be showed that from 60 of 62 were correctly identified. Figure 7, the receiver operating characteristic (ROC) curve for the allimportant features is the value 0.9941 . ROC with the maximum value is 1 , so the ROC values obtained in this study are very high.

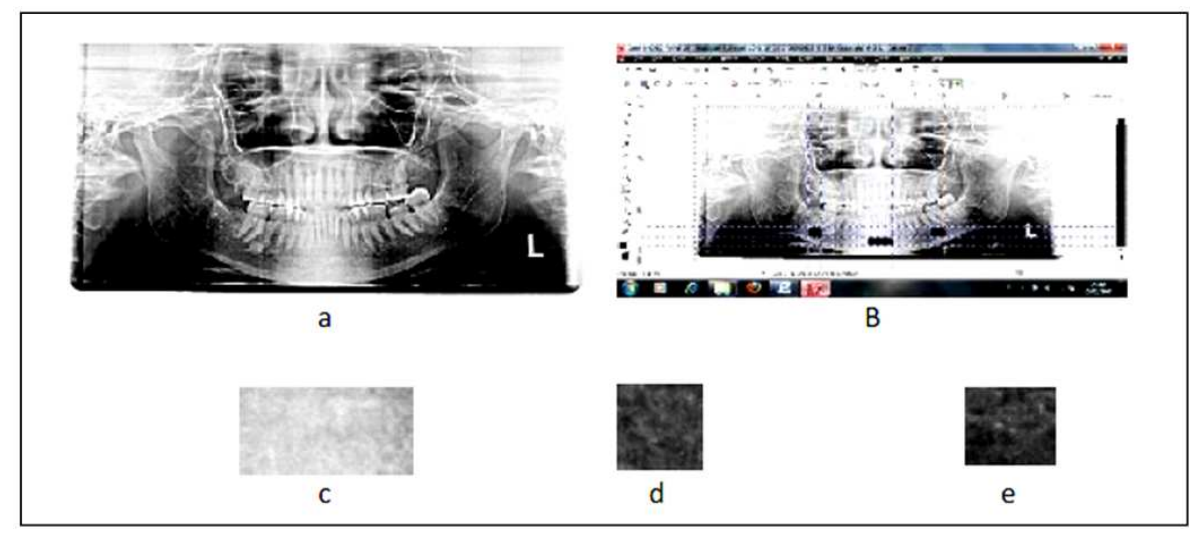

Fig. 2: (a) Panoramic radiograph, (b) selecting RoI (c) anterior RoI image, (d) right posterior RoI image, (e) left posterior ROI image (Widyaningrum and Lestari, 2013)

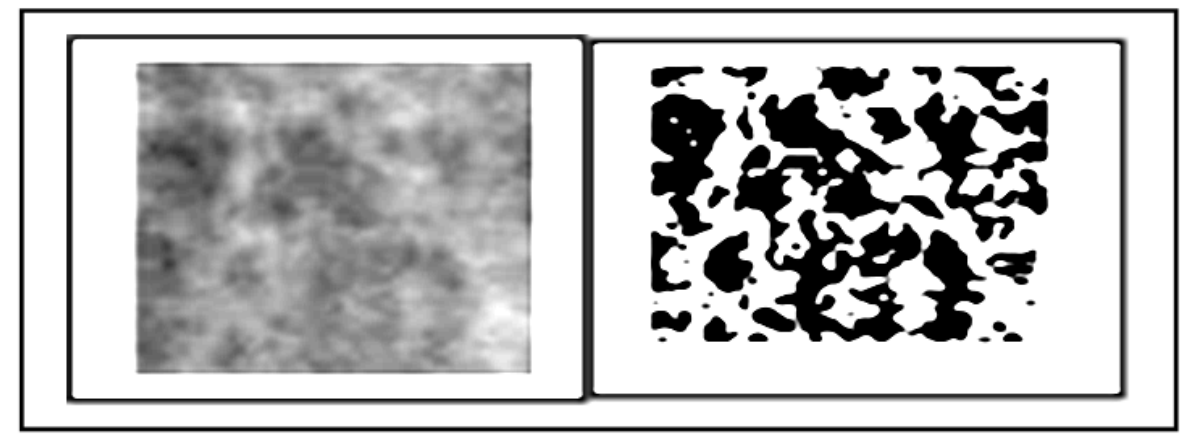

Fig. 3: Example of gray scale and binary RoI 


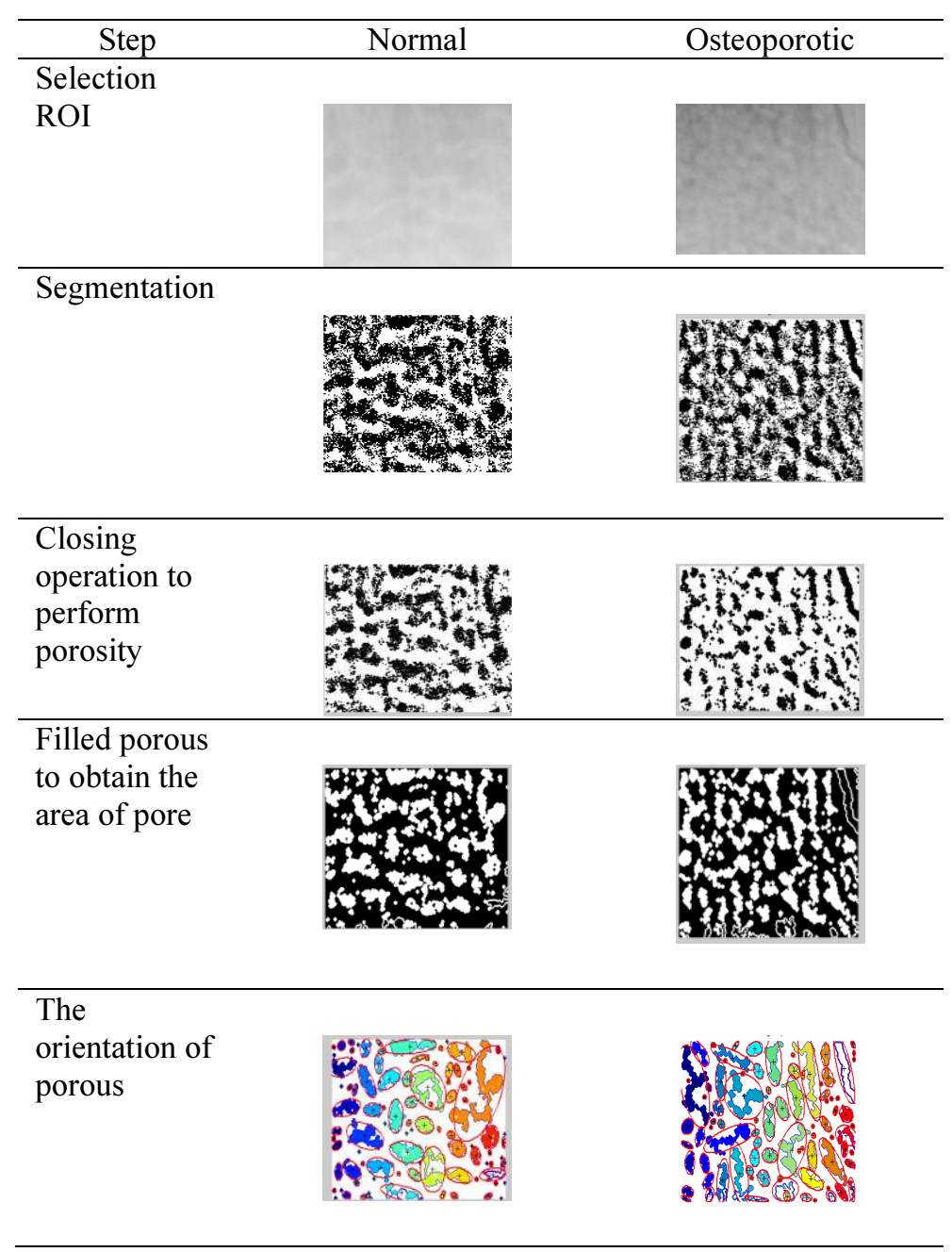

Fig. 4: Porous morphology on normal and osteoporotic class (Sela et al., 2015)

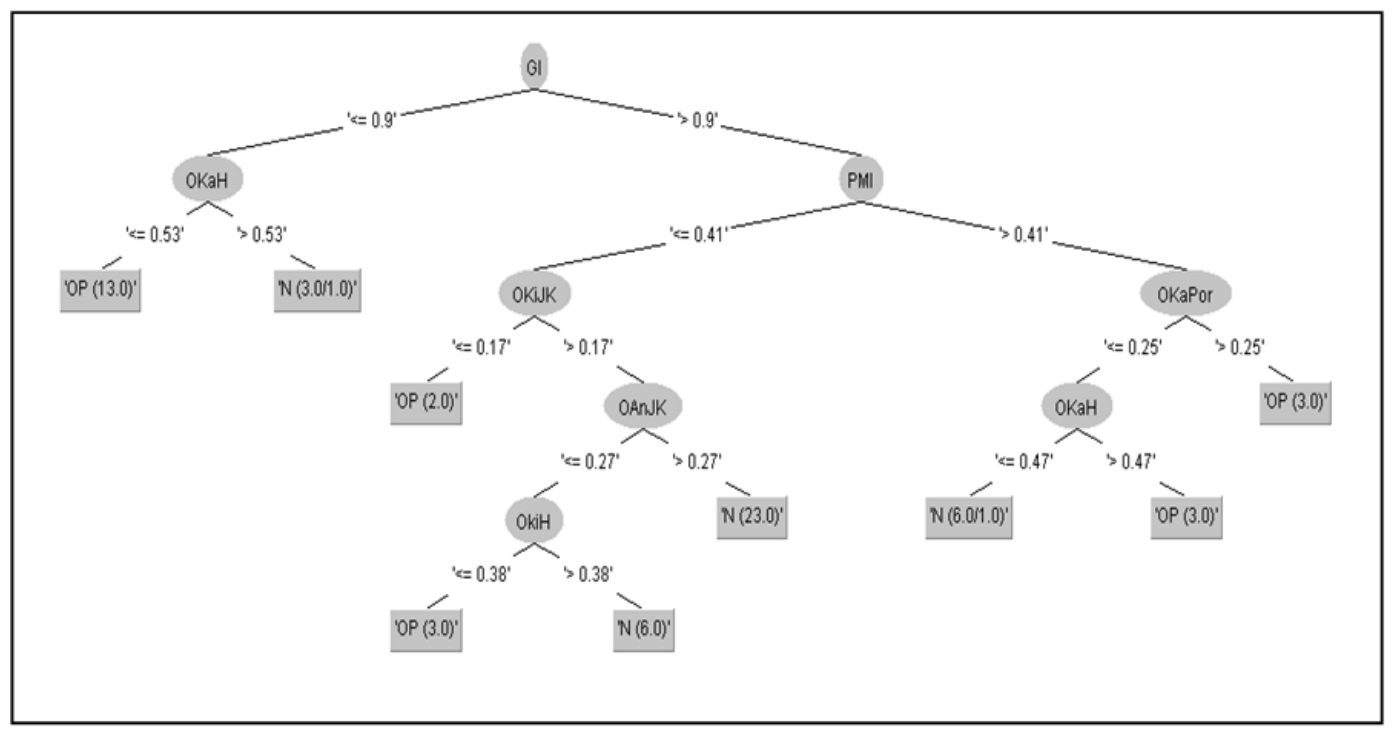

Fig. 5: Decision tree of RMI measurement, texture analysis and morphology analysis features 
Correctly Classified Instances $6096.7742 \%$

Incorrectly Classified Instances $23.2258 \%$

$===$ Confusion Matrix $===$

a b <-- classified as

$360 \mid a=N$

$224 \mid \mathrm{b}=\mathrm{OP}$

Fig. 6: Confusion matrix of RMI measurement, texture analysis and morphology analysis features

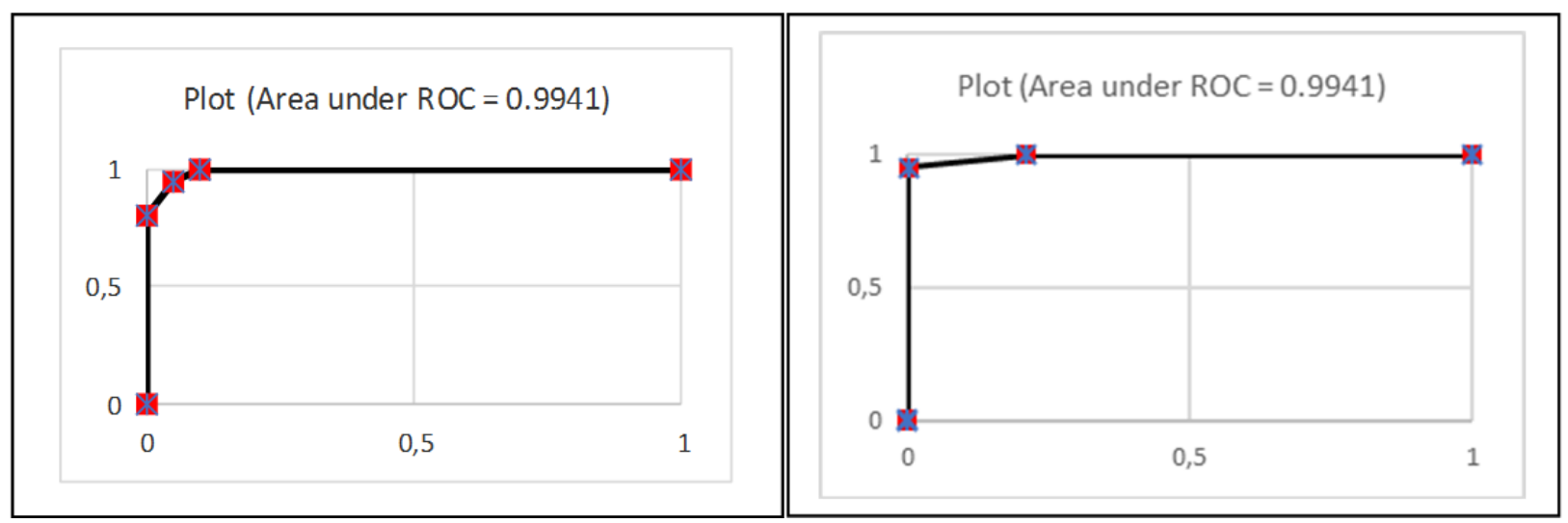

Fig. 7: ROC of RMI, texture analysis and morphology analysis features

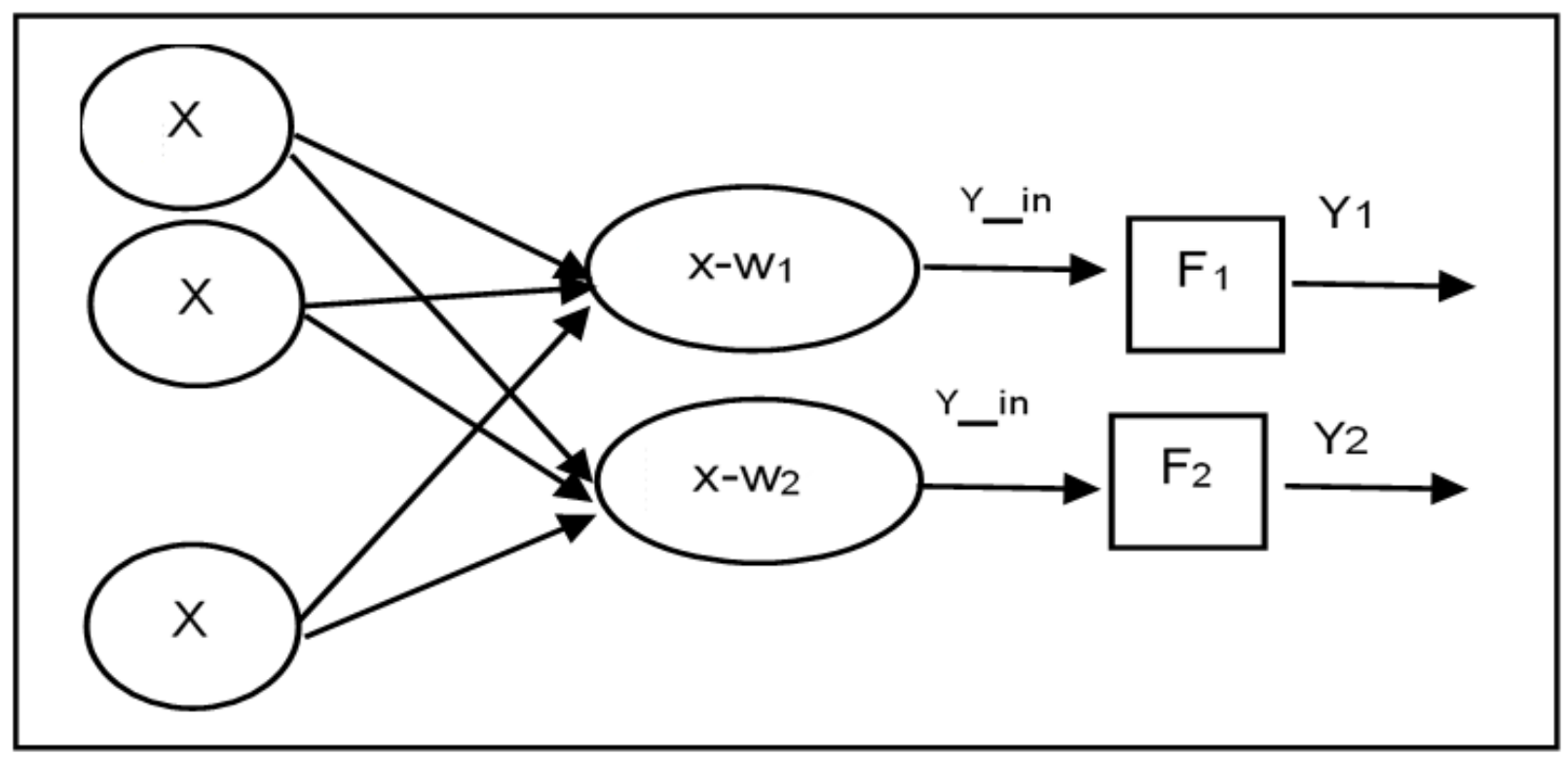

Fig. 8: LVQ architecture 
Subsequently, the selected features (7 features) were trained and tested using LVQ neural networks. Thus, the LVQ architecture is 7 nodes $\left(\mathrm{X}_{1}, \mathrm{X}_{2} \ldots \mathrm{X}_{7}\right)$ on the input layer and 2 nodes $\left(Y_{1}, Y_{2}\right)$ on the output layer (Fig. 8).

The processing that occurs in each neuron is to find the distance between an input vector to the weights (w1 and w2). w1 is the weight vector that connects each neuron in the input layer to the first neuron in the output layer, where w2 is the weight vector that connects each neuron in the input layer to the second neuron in the output layer.

The activation function of $F_{1}$ will map $\mathrm{y}_{-}$in ${ }_{1}$ to $\mathrm{y}_{1}=1$ if $\left|\mathrm{x}_{\mathrm{i}}-\mathrm{w}_{1}\right|<\left|\mathrm{x}_{\mathrm{i}}-\mathrm{w}_{2}\right|$ and $\mathrm{y}_{1}=0$ otherwise. Similarly, the activation function $\mathrm{F}_{2}$ will map $\mathrm{y}_{-} \mathrm{in}_{2}$ to $\mathrm{y}_{2}=1$ if $\left|\mathrm{x}_{\mathrm{i}}-\mathrm{w}_{2}\right|$ $<\left|\mathrm{x}_{\mathrm{i}}-\mathrm{w}_{1}\right|$ and $\mathrm{y}_{2}=0$ otherwise. Testing on 10 images results indicate that LVQ can identify osteoporosis. The parameters used for learning is the learning rate (alpha) $=0.1$, the minimum value of alpha $=0.0001$. The accuracy rate is $80 \%$

\section{Discussion}

Osteoporosis is a metabolic disease characterized by bone decreases in density and increases in porosity. It is resulting in decrease in both trabecular and cortical bone mass (Milliuniene et al., 2008). The porosity of bone is influenced by gender, race, age, and geographic location (Rao et al., 2011).

In previous study (Widyaningrum et al., 2012) femoral neck and lumbar spine $\mathrm{T}$-score values were assessed with DEXA examination, while RMI measurements of MI, PMI, AI and GI were performed on the dental panoramic radiographic images. Pearson correlation showed significant correlation between both of femoral neck T-score and lumbar spine T-score with RMI stated that MI $(\mathrm{p}<0.05, \mathrm{r}=0.443-0.436)$, AI $(\mathrm{p}<0.01, \mathrm{r}=0.526-0.571$ and $\mathrm{GI}(\mathrm{p}<0.01, \mathrm{r}=0.628$ 0.632). Similarly, Spearman correlation test between femoral neck T-score and lumbar spine T-score with PMI showed also significant correlation $(\mathrm{p}<0.05, \mathrm{r}=$ $0.365-0.433$ ). The results of that study suggest that osteoporosis may affect mandibular bone density as indicated declining RMI which is equal to the decline on T-score of femoral neck and lumbar spine.

In Widyaningrum and Lestari (2013), a dental panoramic radiographic image and scanning using DEXA for each site of right femoral neck and lumbar spine were taken from subjects. The aim of this research is to determine the correlation between texture parameter values of mandible trabecular bone with bone mass density values. Texture parameter extraction on ROI of the dental panoramic radiographic has been performed using run length method, i.e. short run emphasis (SRE) and long run emphasis (LRE). SRE and LRE were correlated with each subject's BMD and T score.

Spearman correlation test showed significant negative correlation between SRE of right posterior ROI with T-score of lumbar spine $(\mathrm{p}<0.05 ;-0.280<\mathrm{r}<-$ 0.257). Pearson correlation test also showed significant negative correlation between LRE of left posterior ROI with the BMD of femoral neck $(r<0.05 ;-0.276<r<-0.249)$.

Most of trabecular texture parameters in that research showed no significant correlation with bone mass density value, therefore mandible trabecular feature extraction methods need to be developed to get best fit mandible density predictor using the dental panoramic radiographic.

Result of the features selection in this research shows that RMI measurement, texture features, and morphologycal features indicated the dominant features for osteoporosis identification. This result almost in line with two previous studies, but these results show a discrepancy with previous studies (Gulsahi et al., 2009; Ledgerton et al., 1999).

The resulted his study is slightly difference from (Sela et al., 2014) that performed backpropagation neural network as classifier on selected morphology porous features of periapical images which were classified into 3 classes (normal, osteoporotic, osteopenia). The accuracy rate had $83.84 \%$.

\section{Conclusions}

Based on results that have been achieved, it can be concluded the following matters:

- The extracted features by combining RMI, texture analysis, and morphology analysis, the featuis study result 34 features .

- The seven major features playing significant role in the osteoporosis identification are gonion index, panoramic mandibular index, horizontal pore on right and left posterior, small pore on left posterior, small pore on anterior, and porosity on right posterior.

- The accuracy of the selection features is $96.7742 \%$ and the value of the ROC is 0.9941 .

- The accuracy rate for LVQ testing is $80 \%$. 


\section{Acknowledgement}

The authors would like to thank Rini Widyaningrum to her valuable helps in this research

\section{Author's Contributions}

Enny Itje Sela: Contributed to research, design, literature review, implementing the proposed algorithm, analyzing the results, compare with other existing algorithms and writing of the manuscript.

Sutarman: Contributed to conceptualization, co analyzing the results, writing of the manuscript, editing and reviewing.

\section{Ethics}

The authors declare that there is no ethical issues that may arise after the publication of this manuscript.

\section{References}

Gulsahi, A., S. Ozden, A.I. Cebeci, N.O. Kucuk and C.S. Paksoy et al., 2009. The relationship between panoramic radiomorfometric indices and the femoral bone mineral density of edentulous patients. Oral Radiol, 25: 47-52.

Hiremath, P.S., H.Y. Iranna and J.D. Pujari, 2007. Classification of squamous cell carcinoma based on color and textural features in microscopic images of esophagus tissues. J. Compt. Sci., 3: 566-573. DOI: $10.3844 /$ jcssp.2007.566.573

Ledgerton, D., K. Horner, H. Devlin and $H$. Worthington, 1999. Radiomorphometric indices of the mandible in a british female population. Dentomaxillofac. Radiol., 28: 173-181.

Levine, J.P., 2016. Identification, diagnosis, and prevention of osteoporosis. http://www.ncbi.nlm.nih.gov/ pubmed/

Mantzaris, D., G. Anastassopoulos, L. Iliadis, K. Kazakos and H. Papadopoulos, 2010. A soft computing approach for osteoporosis risk factor estimation. IFIP International and computer scieces. Federation Information Processing AICT 339, pp: 120-127.

Milliuniene, E., V. Alekna, V. Peciuliene, M. Tamulaitiene and R. Maneliene, 2008. Relationship between mandibular cortical bone height and bone mineral density of lumbar spine. Stomatologija, Baltic Dental Maxillofacial J., 10: 72-75.
Rao, G.S., L. Chatra and P. Shenai, 2011. Evaluation of adult cortical bone mass as measured by panoramic mandibular index - a radiographical study. WebmedCentral Radiology, 2: WMC001447.

Sela, E.I., S. Hartati, A. Harjoko, R. Wardoyo and M. Mudjosemedi, 2015. Feature selection of the combination of porous trabecular with anthropometric features for osteoporosis screening, Int. J. Electrical Computer Eng. DOI: 10.4197/Med

Sela, E.I. and R. Widyaningrum, 2015. Osteoporosis detection using important shape-based features of the porous trabecular bone on the dental X-ray images. Int. J. Advanced Computer Science Applications. DOI: 10.14569/IJACSA

Sela, E.I., S. Hartati, A. Harjoko, R. Wardoyo and M. Mudjosemedi, 2014. Shape-based features extraction model of the porous trabecular bone on the dental periapical radiograph images for osteoporosis screening, Unpublished dissertation in partial fulfillment of the requirements for the degree of Doctor of Computer Science, Universitas Gadjah Mada, Yogyakarta, Indonesia.

Sela, E.I., S. Hartati, A. Harjoko, R. Wardoyo and M. Mudjosemedi, 2013. Segmentation on the dental periapical x-ray images for osteoporosis screening. Int. J. Advanced Comput. Sci. Applications.

DOI: 10.14569/IJACSA. 2013.040720

Sutarman, M., A. Majid and E.I. Sela, 2017. Performance evaluation of combined consistencybased subset evaluation and artificial neural network for recognition of dynamic Malaysian sign language. J. Theoretical Applied Information Technology.

Vasudevan, P., 2014. Iterative dichotomiser-3 algorithm in data mining applied to diabetes database. J. Comput. Sci., 10: 1151-1155. DOI: $10.3844 /$ jssp.2014. 1151-1155

Veluchamy, M., K. Perumal and T. Ponuchamy, 2012. Features extraction and classification of blood celss using artificial neural network. Am. J. Applied Sci., 9: 615-619. DOI: 10.3844/ajassp.2021.615.619

Widyaningrum, R., N. Kertia and A. Harjoko, 2012. The relationship between bone mass density and radio morphometry index on menopausal women from Javanese ethnic in Indonesia: a pilot study. Proceeding of The 7th International Conference on Biomedical Engineering and Medical Applications (ICBEMA), Nov. 9-10, BME Days, Serpong-Indonesia. 
Widyaningrum, R. and S. Lestari, 2013. The correlation between mandible trabecular texture parameter on panoramic radiograph with bone mass density, Proceeding of The International Symposium of Advanced Clinical Approach for The Prevention of Dental Caries and Implicated Diseases, Yogyakarta, Indonesia, pp: 206-214.

DOI: $10.2215 /$ CJN.04151206 